\title{
AC 2007-2723: IDENTIFYING FACTORS AFFECTING PERSISTENCE RATES AMONG UNDERGRADUATE ENGINEERING STUDENTS FROM UNDERREPRESENTED POPULATIONS AT THE UNIVERSITY OF VIRGINIA
}

\section{Carolyn Vallas, University of Virginia}

Carolyn Vallas is General Faculty and Director of Center for Diversity in Engineering at the University of Virginia School of Engineering and Applied Science. She received the B.A. degree in Education from Seattle University, and the M.S. degree in Education Psychology and School Counseling from California State University, Fullerton. She is working toward her Ph.D. at the Curry School of Education at the University of Virginia. Carolyn is currently the president of the National Association of Multicultural Engineering Program Advocates. (NAMEPA) and faculty advisor and member for the student chapters of Society of Women Engineers, (SWE), Society of Hispanic Professorial Engineers (SHPE), and the National Society of Black Engineers (NSBE). She is a member of the Women in Engineering Programs and Advocates Network (WEPAN) and ASEE. Her research interest is the education of the underrepresented population in the STEM fields, focusing on teachers and students preparation, retention and persistence factors.

\section{Susan Donohue, University of Virginia}

Susan Donohue is adjunct faculty in the Department of Systems and Information Engineering in the School of Engineering and Applied Science at the University of Virginia. She will serve as an AGEP Postdoctoral Engineering Education Researcher (PEER) through the Center of Advancement in Engineering Education Research (CASEE), the National Academy of Engineering starting in May, 2007. She received the B.A. degree in political science from Marquette University, Milwaukee, Wisconsin and the M.E. and Ph.D. degrees in systems engineering from the University of Virginia, Charlottesville. Her academic honors include memberships in Phi Beta Kappa, Alpha Sigma Nu, and Omega Rho. Her professional affiliations include IEEE and ASEE. Her main research interests include knowledge engineering and retention/persistence issues. 


\title{
Identifying Factors Affecting Persistence Rates Among Undergraduate Engineering Students from Underrepresented Populations
}

\begin{abstract}
The literature abounds with descriptions of factors affecting persistence rates both positively and negatively among undergraduate engineering students from underrepresented populations. For the purposes of this study, the relevant underrepresented populations are African American, Hispanic American, and Native American. Some of these factors universally affect all students, and others are specific to a school of engineering's culture. We propose a survey to identify the factors most significantly affecting persistence rates at the School of Engineering at the University of Virginia (U.Va. SEAS), the developmental form of which is presented in this paper, as the first step in creating retention programs that will work effectively in our institutional culture.
\end{abstract}

Keywords: Persistence, retention, underrepresented populations in engineering

\section{Introduction}

Research into the factors affecting persistence among undergraduate engineering students from underrepresented populations addresses a critical need. There is a well documented gap between the demand for technically educated professionals needed to maintain the United States' competitiveness in science and engineering and the supply graduating from U.S. schools and colleges of engineering. ${ }^{1}$ Overall, domestic demand for engineers and computer scientists is predicted to rise by $36 \%$ by 2010 , yet the number of engineering baccalaureate degrees granted by colleges and universities in the U.S. was off in 2004 by $20 \%$ from the peak number in 1985 . And the national retention rate in engineering, as of 2006, is $48 \%$, a rate that has remained relatively flat for over a decade. ${ }^{2}$ No student population demographic can be neglected in the effort to close the supply-demand gap. Historically, certain populations - African American, Hispanic American, and Native American - are underrepresented in engineering in proportion to their numbers in the general population due to many cultural, social, and academic factors. (see, for example, ${ }^{3}$ ) These populations also represent "the largest untapped resources available to help maintain and/or increase engineering enrollments" " although they, regrettably, enroll in engineering studies in smaller numbers and leave in larger numbers than students from other populations. ${ }^{5}$ Consider, too, the fact that it is more cost effective to retain undergraduate engineering students than to recruit them. ${ }^{6}$ It is therefore imperative to identify and incorporate the factors most affecting persistence - both "leaving" and "staying" factors - among students from underrepresented populations into retention initiatives in order to increase the probability of the initiatives' success.

There are several ways to elicit information regarding the factors most significantly affecting persistence rates: observation, interviews, focus groups, and surveys. We choose to start with a survey for several reasons. First, a survey is the most efficient means for quickly collecting a standardized set of data that can be used to structure future research efforts. Second, the survey will purposefully support a mixed methods (a combination of quantitative and qualitative 
methods) research design. The quantitative data will help us develop generalizable conclusions, and the qualitative data will help us to identify factors influencing persistence at the individual level. Finally, we would like to build on the results of a survey conducted by the Engineering Student Council (E-Stud) in Fall, 2006, at the request of the School of Engineering and Applied Science (SEAS) Dean's office, to determine why respondents left SEAS. We are interested in determining how the persistence factors cited by the literature play out at the University of Virginia (U.Va.) as well as identifying the persistence factors unique to our institution's culture so we may make the most effective recommendations regarding retention initiatives.

First, a clarification of terms. A student persists in a course of study; a program retains a student. The focus of the literature on retention presents research and findings from the programmatic point of view; the focus of the literature on persistence studies the factors influencing a student to remain in a chosen discipline. Regardless of the point of view, there are basic factors affecting student persistence/retention in undergraduate engineering studies: self confidence, ${ }^{7,8,9}$ attitudes towards engineering, ${ }^{9,10}$ quality of instruction, ${ }^{11,12}$ and the quality of the college experience. ${ }^{10}$ Additional factors influencing the persistence of students from underrepresented populations include feelings of isolation and perception of a hostile environment. ${ }^{5}$ The main predictors of success in engineering studies, grades (high school, for first year students and core courses, for first and second year students) and SAT scores, are not to be confused with predictors for persistence, for a student does not need a high GPA to remain in engineering studies. ${ }^{7,11,13}$ For that reason, studies that conclude that predictors of academic success are important predictors of persistence (see, for example, ${ }^{6}$ ) should be viewed critically to ensure that confounding variables are not considered in analysis.

\section{Survey Development}

The proposed survey's development is guided primarily by two sources. The first source is the findings from the aforementioned SEAS E-Stud "leavers" survey, which provide partial confirmation of "leaver" factors cited in the literature. The E-Stud survey will also help us to identify "specific to U.Va." factors. The second source is results from a review of the pertinent literature. Citations such as ${ }^{9}$ which provide sample validated survey questions are very helpful in the development process.

There are two intended audiences for the proposed survey. The first audience is current SEAS undergraduate engineering students from underrepresented populations; we would like to identify factors supporting their persistence (i.e., why are they "stayers"). The second is former SEAS undergraduate engineering students from underrepresented populations; we would like to identify the factors that led to their leaving the study of engineering (i.e., why are they "leavers").

\section{E-Stud Survey Results}

The majority of respondents to the E-Stud survey ( $n$ not specified but is assumed to be a minimum of 49 , based on the number of responses to a question eliciting "other reasons not mentioned") cited lack of flexibility in the curriculum (73.6\%) as the main reason they left SEAS. An analysis of responses on this topic indicated that the respondents may have been 
likely to stay in engineering if the curriculum had been flexible enough to allow them more opportunities to take courses in the humanities and social sciences. Other significant responses are:

- the type of work or concentrations offered were not a good fit

- the school was different from what was expected

- the school did not meet expectations

- lack of personal attention

- required courses irrelevant to ultimate goal

- lack of support for ANY (emphasis students') creativity

No demographic information about the respondents was provided, so we cannot determine "leaving" factors specific to students from underrepresented populations from the survey responses.

\section{Survey Questions}

The demographic data will help us stratify and analyze responses by race, gender, economic, and academic class.

Tell us about yourself. While we would appreciate answers to all of the questions, answering them is optional.

I am a

I gender identify as

My main ethnic identification is

My education is funded by

I self identify my family as

If you left SEAS, please indicate when:

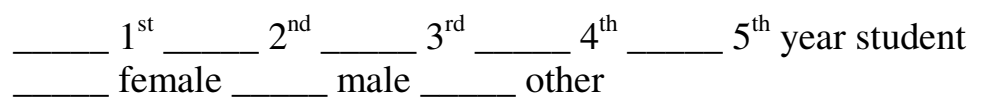
African American Native American $\%$ me _ $\%$ parent/guardian "blue collar"__ "white collar" but not executive executive level other Hispanic American class year semester

A Likert scale of $1=$ strongly disagree to $5=$ strongly agree will be used to elicit answers to the following questions.

\section{Self Confidence}

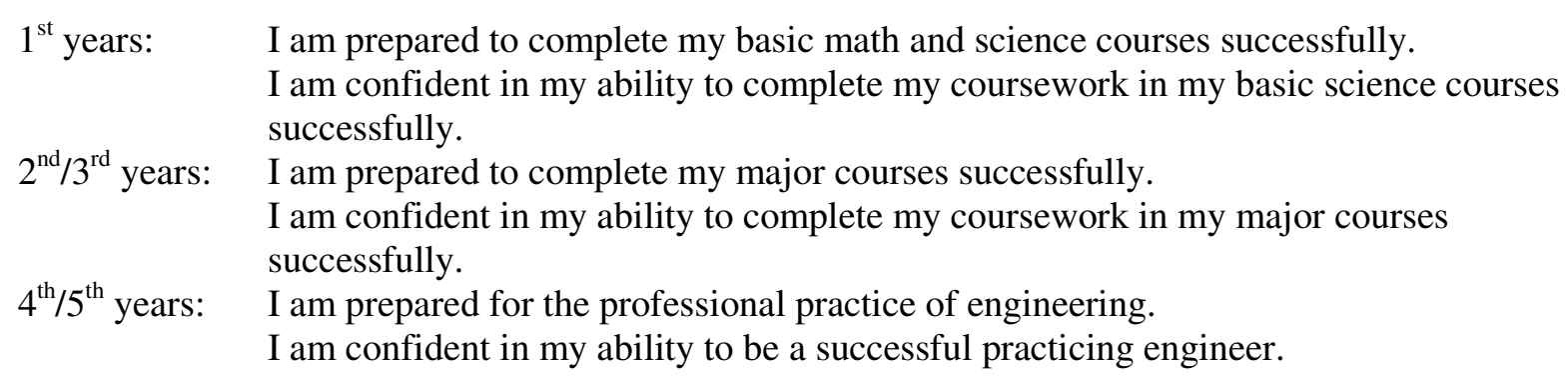

All: I am secure in my abilities to succeed academically.

I am secure in my abilities to succeed personally. 


\section{Attitudes Towards Engineering}

I enjoy the study of engineering.

I study engineering only because I want a good job when I graduate.

I have a positive awareness of the general practice of engineering.

I have a positive awareness of the practice of engineering in my (intended) major.

\section{Quality of Instruction}

My professors in the following courses were competent: in my core courses, overall in my major courses, overall in my electives, overall in my Science, Technology, and Society (STS) courses; repeat for teaching assistants

My professors in the following courses were caring: in my core courses, overall in my major courses, overall in my electives, overall in my STS courses; repeat for teaching assistants

I basically had to teach myself in the following courses: in my core courses, overall in my major courses, overall in my electives, overall in my STS courses

\section{Quality of College Experience}

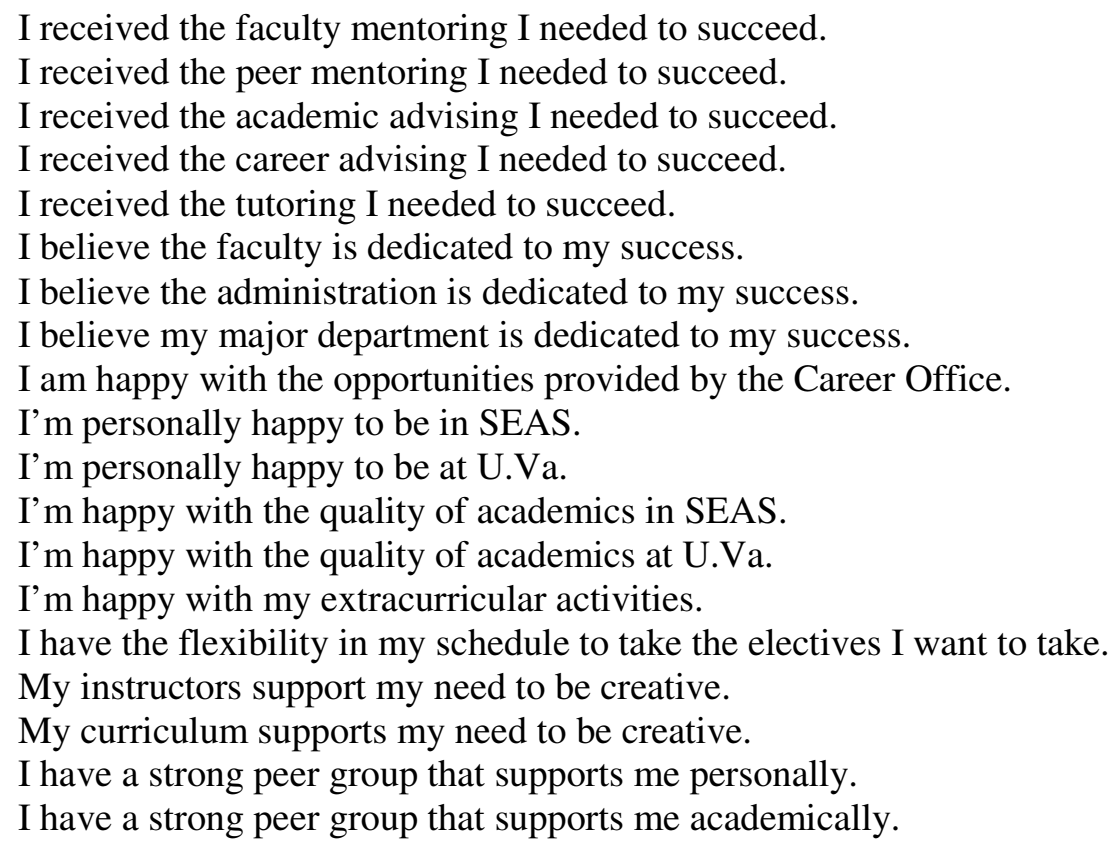

Finally, we will provide respondents the opportunity to add structured and free-form comments and observations. These qualitative data will be analyzed to determine additional factors affecting persistence among our target populations. The results will also be used to formulate questions for follow-up through interviews and focus groups. The questions are listed on the following page. 


\section{Initial Survey Validation}

This version of the survey was subjected to both face and content validation by its developers. "Face validation," or the physical appearance of validity, is assessed by evaluating whether the instrument in question is believed to capture the intended information. "Content validation" refers to the instrument's ability to provide a representative sample of the subject matter under study. ${ }^{14}$ Based on our experience and familiarity with similar surveys, we believe that the survey questions will elicit the expected data.

For those who stayed in engineering: what are the major influences or factors keeping you in engineering? What, if anything, would make you leave the study of engineering?

If you stayed at U.Va., what were the major influences or factors keeping you here? If you transferred to study engineering another university, why did you transfer?

For those who left engineering: what were the major influences or factors in your decision to leave engineering? What, if anything, would have made you stay in engineering?

If you stayed at U.Va., what were the major influences or factors keeping you here? If you transferred to another university, why did you transfer?

For all: would the opportunity to participate in research as an undergraduate have any influence on your decision?

Do you anticipate working in engineering or a related profession after graduation?

If so, for how long? If you plan a short career ( $>5$ years), to which career path do you plan to switch?

If not, in what field do you plan to work? Will you need graduate education to accomplish your career goal?

Any other comments? Thank you so much for your honest responses!

\section{Future Work}

Future work includes the piloting, modification, and further validation of this survey; administration and analysis of this survey, including obtaining IRB approval; the extension of the survey to identify factors influencing persistence among graduate engineering students from underrepresented populations; and performing principal components analysis (PCA), as the data warrant, to determine the most influential factors to evaluate. The ultimate outcome is the development and implementation of initiatives to support the influence of positive factors and ameliorate the effects of negative factors. With the information provided by the survey, we will be able to build institution-specific programs based on proven retention strategies such as cooperative learning ${ }^{15,16,17}$ and learning communities, ${ }^{18}$ but tweaked to our institutional culture and the needs of our students from underrepresented populations. We will also use the 
information to strengthen and expand our popular high school to $1^{\text {st }}$ year bridge, or transition, program in both numbers and in years covered (i.e., $1^{\text {st }}$ to $2^{\text {nd }}$ year and $2^{\text {nd }}$ to $3^{\text {rd }}$ year BRIDGEs).

We will enlist the help of the local chapters of the National Society of Black Engineers (NSBE) and the Society of Hispanic Professional Engineers (SHPE) in survey administration.

\section{Acknowledgments}

We thank the reviewer for his/her comments, the SEAS Dean's Office for permission to use the referenced survey results, and the students and staff of the Center for Diversity in Engineering for their dedication.

\section{References}

1. Committee on Sciences, Engineering, and Public Policy, National Academies of Science and Engineering, and the Institute of Medicine. Rising Above the Gathering Storm: Energizing and Employing America for a Brighter Economic Future. Washington, DC: The National Academies Press, 2006. (pre-publication version available at http://www.nap.edu/catalog/11463.html, accessed in 9/06)

2. Grose, T.K., "Booting Up,” PRISM: American Society for Engineering Education, 16(1), September, 2006, pp. $26-31$.

3. Chubin, D.E; May, G.S.; and Babco, E.L., "Diversifying the Engineering Workforce," Journal of Engineering Education 94(1), January, 2005, pp. 73 - 86.

4. Anderson-Rowland, M.R., "A First Year Engineering Student Survey to Assist Recruitment and Retention," Proceedings of the $26^{\text {th }}$ Annual Frontiers in Education Conference (1996), Volume 1, pp. 372 - 376.

5. Hein, G. and Monte, A., "A Student Mentoring and Development Program for Underrepresented Groups in Engineering," Proceedings of the $34^{\text {th }}$ Annual Frontiers in Education Conference (2004), Volume 3, pp. S1G/4 S1G/8.

6. Acker, J.C.; Hughes, W.; and. Fendley, W.R. Jr., "Implementing a Recursive Retention Assessment System for Engineering Programs," Proceedings of the Association for Institutional Research (2002).

7. Felder, R.M.; Felder, G.N.; and Dietz, E.J., “A Longitudinal Study of Engineering Student Performance and Retention. V. Comparisons with Traditionally-Taught Students," Journal of Engineering Education 87(4), October 1998, pp. $469-480$.

8. Astin, A.W., "Engineering Outcomes," PRISM: American Society for Engineering Education, September 1993, pp. 27-30.

9. Besterfield-Sacre, M.; Atman, C.J.; and Shuman, L.J., "Characteristics of Freshman Engineering Students: Models for Determining Student Attrition in Engineering," Journal of Engineering Education 86(2), April, 1997, pp. $139-149$.

10. Burtner, J., "Critical-To-Quality Factors Associated With Engineering Student Persistence: The Influence of Freshman Attitudes," Proceedings of the 34 ${ }^{\text {th }}$ Annual Frontiers in Education Conference (2004), Volume 2, pp. F2E-1 - F2E5.

11. Seymour, E. and Hewitt, N.M. Talking About Leaving: Why Undergraduates Leave the Sciences. Boulder, Colorado: Westview Press, 1997. 
12. Felder, R.M. and Brent, R., "Understanding Student Differences," Journal of Engineering Education 94(1), January, 2005, pp. $57-72$.

13. Levin, J. and Wyckoff, J., "Findings - Effective Advising: Identifying Students Most Likely to Persist and Succeed in Engineering," Engineering Education 78(11), 1988, pp. 178 - 182.

14. Krathwohl, D.R., Methods of Educational and Social Science Research: An Integrated Approach (2 Edition), Long Grove, Illinois: Waveland Press, 1998.

15. Howard, B.C., Learning to Persist-Persisting to Learn (Revised Edition), Washington, DC: Mid-Atlantic Equity Center, American University, 1989.

16. Smith, K.A.; Sheppard, S.D.; Johnson, D.W.; and Johnson, R.T., "Pedagogies of Engagement: ClassroomBased Practices," Journal of Engineering Education 94(1), January, 1995, pp. 87 - 101.

17. Williams, L. "Debunking the Nerd Stereotype with Pair Programming," IEEE Computer 39(5), May, 2006, pp. $83-85$.

18. Blat, C. and Tolley, P., "Maximizing Academic and Professional Success: Building Student Learning Communities That Lead to Engineering Excellence," Proceedings of the $111^{\text {th }}$ American Society for Engineering Education Annual Conference and Exposition (2004). 